\title{
Fertilité et Obésité
}

\section{Introduction}

\section{R. Lévy · M. Pigeyre}

(C) Lavoisier SAS 2017

Ce numéro thématique, particulièrement riche, nous propose des synthèses pertinentes sur l'impact du surpoids et de l'obésité sur la fertilité des couples, une des comorbidités fréquentes de l'obésité, encore insuffisamment comprise et prise en charge.

La prévalence de l'obésité a triplé ces trente dernières années chez les femmes comme chez les hommes en âge de procréer, et coïncide avec l'augmentation de l'infertilité qui touche actuellement un couple sur six en France.

Chez les femmes, le surpoids et l'obésité constituent un facteur non négligeable de baisse de la fertilité naturelle : troubles de l'ovulation, augmentation du délai nécessaire pour concevoir, diminution des chances de succès des traitements et augmentation du risque de fausses couches. Une prise en charge hygiéno-diététique pré-conceptionnelle doit être mise en place précocement, à la fois pour améliorer la fertilité, mais aussi pour diminuer les futures complications obstétricales.

Chez les hommes, de nombreux facteurs concernant le mode de vie et l'environnement sont susceptibles d'agir sur la fertilité en provoquant des altérations spermatiques, aussi bien en termes de quantité que de qualité. Parmi ces facteurs, l'état nutritionnel, le poids et le statut métabolique seraient associés à l'infertilité. Plusieurs travaux ont également été menés sur l'implication potentielle des adipokines sur les fonctions de reproduction.

La chirurgie bariatrique constitue un traitement efficace de l'obésité sévère permettant une perte de poids conséquente. Chez les femmes, elle semble avoir un effet positif sur la régulation des cycles menstruels et sur l'ovulation. Chez les hommes, elle permettrait une amélioration de l'hypogonadisme hypogonadotrope, mais ses effets sur les paramètres spermatiques semblent controversés.

Le dernier chapitre de ce dossier est consacré à la contraception des femmes obèses. Il est maintenant établi que les femmes obèses ont une activité sexuelle, non différente des femmes de poids normal, alors qu'elle bénéficieraient d'une couverture contraceptive moindre et d'un risque de grossesse non désirée plus élevé. L'obésité constitue par ailleurs un important facteur de risque d'accidents thromboemboliques veineux et artériel. L'ensemble de ces facteurs est donc à intégrer dans le choix d'une méthode contraceptive chez une femme obèse.
R. Lévy

Sorbonne Universités, UPMC Univ Paris 06, Centre de recherché

Saint Antoine, INSERM US938, 75012 Paris, France

AP-HP, Hôpital Tenon, service de biologie de la reproduction CECOS, Paris, France

\section{Pigeyre ( $\square)$}

Service de nutrition, Hôpital Claude Huriez, CHRU de Lille, av. Oscar Lambret, 59037 Lille cedex, France

e-mail : marie.pigeyre-2@univ-lille2.fr 